\title{
BRAVE: Beacon-less Routing Algorithm for Vehicular Environments
}

\author{
Pedro M. Ruiz, Victor Cabrera, Juan A. Martinez, Francisco J. Ros \\ Dept. of Information and Communications Engineering \\ University of Murcia, Spain E-30100 \\ Email: pedrom@um.es, victorcabrera@um.es, juanantonio@um.es, fjros@um.es
}

\begin{abstract}
We study the problem of multihop routing in vehicular ad hoc networks (VANET). IEEE 802.11p and other vehicular network standards advocate vehicles to issue periodic broadcast messages at regular intervals called beacons. Beacons include among other information geographic coordinates of the vehicle, heading, speed, etc. Thus, most VANET routing solutions in the literature use those beacons to know available neighbors and take position-based routing decisions. However, we argue that using that information to take routing decisions can result in inefficiencies such as temporal loops in the forwarding path, backward progress due to stale information and use of lowquality links. We propose a new protocol called BRAVE in which neighbor selection is done opportunistically in collaboration with neighbors. Our simulation results show that BRAVE is able to outperform existing solutions in a realistic urban scenario for a variety of network densities.
\end{abstract}

\section{INTRODUCTION}

Vehicular Ad hoc Networks (VANET) have emerged as a high-potential technology to enable new networking environments. They have gained a lot of research interest during the last years both in industry and academia. They consist of a set of vehicles equipped with wireless interfaces that allow direct communication among them. By using multihop communications vehicles can send messages to other vehicles or devices located outside the radio range of the sender.

The process of sending a message to one or multiple destinations located several hops away from the sender in this context is known as VANET routing. The role of VANET routing protocols is finding a path (list of vehicles) connecting the source to the destination. Although the problem of multihop routing has been widely studied in the context of mobile ad hoc networks (MANET), vehicular networks have very distinctive properties for which MANET routing protocols are not suitable. For instance, nodes follow a restricted mobility pattern because vehicles can only move along roads. Traffic lights, intersections and the likes, produce topologies with highly uneven network densities and frequent partitions. Finally, vehicles have much lighter energy efficiency requirements. All these fundamental differences have made the research community to focus on VANET-specific routing solutions.

In the last few years, we have seen a large number of VANET routing solutions ( [1], [2]) coming up. Their operation can be classified according to multiple criteria. In our case, we comment on the most well-known solutions according to the type of information used by the routing protocol to take routing decisions. The most simple schemes such as Greedy Parameter Coordinator Routing (GPCR [3]) and Connectivity-Aware Routing (CAR [4]) work only with information obtained via control messages received by other vehicles. The adapt the concepts of geographic routing to the VANET environment. In both protocols, the key idea is to forward messages along streets and take routing decisions when messages reach intersections.

Other protocols such as Greedy Source Routing (GSR [5]) and Spatially-Aware Routing (SAR [6]) assume that vehicles are equipped with maps of the cities, and use that extra information to assist the routing protocol. The idea is to apply Dijkstra's shortest path algorithm over the streets of the map to find a route to the position of the destination. Then greedy routing is used to move along the selected streets. Unlike GSR, SAR introduces three recovery strategies to deal with the case in which the selected route has not enough density of vehicles for the routing to proceed.

There are some other solutions such as GyTAR [7], ASTAR [8], MDDV [9], VADD [10] and SADV [11] that assume not only the use of maps, but also information about the traffic along the different streets. The goal is selecting paths with enough vehicles for the routing to progress. In particular, GyTAR uses traffic density information to decide the best street after reaching an intersection. A-STAR gives priority to streets traversed by bus lines. Similarly, MDDV uses the number of lines in a street to weight the importance of that particular street. The latter two schemes (VADD and SADV) incorporate delay into their decisions. VADD estimates the delay to traverse streets (either through multihop forwarding or by any of the cars carrying the message). SADV introduces fixed nodes in each junction to store the messages until a vehicle moving along the desired street passes along.

Finally, there are other solutions such as MOVE [12] or GeOpps [13] in which routing decisions are taken based on the trajectories of neighboring vehicles. Each vehicle carries data messages and each message is only forwarded to a neighbor if its estimated trajectory is better according to some routing metric. For instance, GeOpps works based on minimizing the estimated time of delivery.

As we described in [14], existing solutions suffer from a number of problems that affect their performance. First of all, selection of next hops based on their progress may end up in failed transmissions and retransmissions. Second of all, the use 
of beacon messages induce forwarding inefficiencies including cycles caused by stale information. Third of all, some of these protocols fail to deliver messages because they are not able to handle disconnected topologies. Finally, for trajectory-based routing schemes objective functions may make data packets to fall into a local optimum until the message is dropped.

In this paper, we present a novel beacon-less routing scheme for VANETs called BRAVE. To avoid the issues commented above, the protocol uses an opportunistic forwarding scheme. That is, the next forwarder for the data message is reactively selected among those neighbors that have successfully received the message. In addition, the protocol is able to operate in a store-carry-and-forward paradigm to deal with uneven network densities and disconnected topologies. Moreover, the proposed solution is fully localized (only needs information provided by neighbors) which guarantees ultimate scalability with respect to the number of vehicles in the network.

The remainder of this paper is organized as follows: In section II, we explain in detail the operation of BRAVE. Section III, shows the results of the performance evaluation of the most relevant routing schemes. Finally section IV draws the main conclusions and comments open issues.

\section{BeACON-Less Routing Algorithm FOR VeHiCUlar ENVIRONMENTS}

Our proposed Beacon-less Routing Algorithm for Vehicular Environments (BRAVE) is a fully-localized protocol specifically designed considering the design issues mentioned above. The overall idea behind its operation revolves around the ideas of spatial awareness and beacon-less geographic forwarding. By spatial awareness we refer to allowing intermediate nodes to change the initial plan (streets to follow) based on their local information and their view of the street map. This allows the protocol to avoid following trajectories which may become bad alternatives as the message travels to the destination. In addition, it avoids having to disseminate additional information (e.g. density of vehicles along streets) across the whole VANET, so that any possible data source can compute a good trajectory. Thus, with our proposed scheme additional information only needs to be disseminated to nearby areas.

Unlike many of the previous solutions, BRAVE performs hop-by-hop data forwarding along a selected street using an opportunistic next hop selection based on the idea of beaconless geographic routing. Instead of taking forwarding decisions based on positions of neighbors gathered by periodic beacons, BRAVE uses a reactive scheme. The current forwarder sends the data packet and the next forwarder is selected among those who already received the data message correctly. To give priority to those providing better improvement, we adjust the timing of the responses so that the best candidates (according to some metric) answer first and cancel responses from other nodes. This idea solves well-known issues in existing routing protocols that we reported in [14].

In the next subsections we elaborate on the detailed operation of each part of the protocol and how they work together to route data messages efficiently in VANETs.

\section{A. Spatial Awareness or Additional information}

The assumption that vehicles have access to a street map and possibly some additional information such as traffic densities, estimation of delays, etc. has become quite common in existing solutions. In most protocols the source vehicle generating the data message computes the shortest path from its current position to the position of the destination along the street map. To do that, they model the street map as a graph where edges represent streets and junctions are represented by vertices. Some solutions use additional information just to add weights to edges before computing the shortest path. Then, some sort of geographic forwarding is used to send data along that path.

In BRAVE, the street map and any available additional information is used to select the next junction to be reached using hop-by-hop forwarding. The main novelty is that in our case the trajectory of the packet is not pre-computed by the source vehicle. Each intermediate vehicle re-computes the trajectory and decides to which next junction the data message is routed. So, changes in the direction the message should follow may happen at any intermediate vehicle with updated information, not only when vehicles or messages reach junctions.

To compute the next junction, the current node holding the data packet applies Dijkstra's shortest path algorithm to the (possibly weighted) street graph. After that, it stores the next two junctions towards the destination. The first one is used as the next intermediate destination for the geographic routing process. The second one is used to help in the process of deciding when to change from one street to another.

In BRAVE, a change from one street to another is done by changing the next intermediate destination to the second junction, and computing as explained above a new second junction. This is done when the current vehicle has any neighbor whose distance to the second junction is smaller than the distance between the two junctions. We can see in Fig. 1 an example in which the distance between the neighboring vehicle $a$ and the second junction is lower than the distance between the two junctions. As shown, this condition is sufficient to guarantee that the street change happens where required. Moreover, this solution works well without needing to rely on any external configuration parameter such as distance to junctions, radio range, etc. which have been proven to generate problems in other protocols.

As we have seen this concept of using two junctions to guide the geographic routing process allows following an overall trajectory without the overhead of carrying the whole trajectory in data packets. In the next subsection we discuss how the data forwarding proceeds towards the first junction using other vehicles as relays.

\section{B. Data forwarding along streets}

In the previous subsection we have analyzed how BRAVE decides to route a data messages along a different street. Now we discuss how a message is routed within the current street. As in most VANET routing schemes we employ a variant of geographic routing so that vehicles forward the message to one 


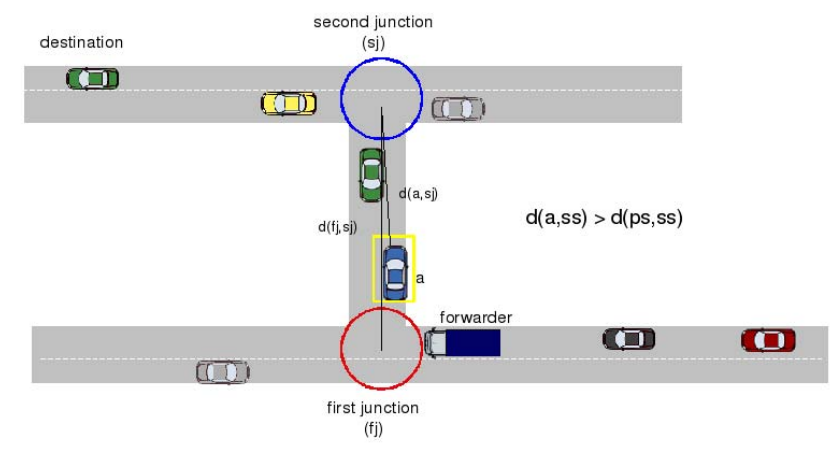

Fig. 1. Illustration of the criteria to change between first and second junction.

of its neighboring vehicles being closer to the next junction then itself. However, as we showed in [14] existing protocols have serious issues to effectively route data messages. In particular, their main issue is that they use periodic beacons to gather positions of neighbors, producing inconsistencies that may lead to stale information, forwarding loops, and so on. Another issue which is often neglected about the use of beacons is that even if information contained in beacons is fully accurate, as beacon messages are small, they usually have higher probabilities of correct reception than data messages. Thus, in realistic deployments it happens oftentimes that a vehicle receives beacons from a neighbor whose radio link is so weak that data messages cannot reach the next hop.

To avoid those issues, our data forwarding strategy is based on beacon-less routing. That is, rather than selecting next hops based on information provided by beacons, BRAVE selects next hops reactively among those nodes that successfully received the data message. The advantage is that this solution prevents failed forwardings due to neighbors for which beacons were received but for which the radio link is quite bad to successfully receive the data packet. In [15] we showed that this idea has been proven very effective to improve geographic routing in realistic wireless sensor network deployments. In this paper, we extend our BOSS protocol to deal with the mobility and characteristics of vehicular networks. The most significant enhancement is the addition of data-ferrying capabilities so that messages can be temporarily stored if there is not a good forwarding opportunity. Also, previously stored messages are considered when new neighbors are discovered.

To avoid the use of beacons, BRAVE uses four message types: DATA, RESPONSE, SELECT and ACK. Given that there may be multiple data sources in the VANET, each of these messages should be clearly identified as belonging to a particular message forwarding. So, they all include what we call a message key, which is just an unique value which is obtained by concatenating the identifier of the source node (SRC) and a sequence number (SEQ) which is set by the source when generating the message. Thus, a forwarding vehicle holding a data message which is being routed along a street broadcasts to its 1-hop neighborhood the DATA message and waits for responses during a certain period of time $T_{\max }$. This DATA message contains the position of the current vehicle as well as the position of the destination and the next two junctions.

Every neighbor receiving the DATA message and being closer to the next junction than the current forwarding node stores this DATA message and schedules a RESPONSE message. The time at which this response message is scheduled depends upon the goodness of this neighbor as next hop. For instance, if our routing metric is hop count, then it is scheduled so that closer the neighbor is to the next junction the sooner it sends back its RESPONSE message. In the next subsection we describe all the details about how to set that time and some improvements to the basic operation to prevent multiple responses.

Once the forwarding vehicle has received the RESPONSE message, it broadcasts a SELECT message that indicates which neighbor is selected as the next forwarder and the message key of the corresponding DATA message. Each neighbor receiving this message checks whether it has been selected as next hop or not. If it has not been selected, then it just deletes the DATA message from its buffer and goes back to its initial state. If it has been selected then that node becomes the forwarder and starts the process again.

To increase the reliability of the protocol, once the SELECT message has been broadcasted the forwarding node schedules a timer waiting for an ACK message. Thus, the last step consists of confirming the reception of the SELECT message by the new forwarding node. In the normal case, the next DATA message forwarding by the next hop serves as an implicit acknowledgment. However, if the new selected next hop is not resending the DATA message (e.g. has no neighbors to forward the message to and temporarily buffers the message) then it must send back an explicit ACK message.

If the previous forwarder does not receive an acknowledgment message it resends the SELECT message up to two additional attempts. If after that, the SELECT message is not acknowledged then the forwarding vehicle restarts the forwarding process. This mechanism ensures that a packet has been delivered from a forwarding vehicle to the next one. As we shall show in our simulation results, this idea of using opportunistic forwarding among nodes that already received the DATA message together with these retransmission schemes avoids the "Range Limit Problem", which we showed to be the main cause of packet drops [14] in many VANET routing solutions.

Unlike traditional geographic routing schemes, BRAVE does not use any recovery scheme to escape from local minima. That is, when a message reaches a vehicle having no neighbors closer to the next junction than itself. The reason is that perimeter mode has been proven to be of very marginal benefit in VANET topologies in which most escaping alternatives are along the same street. To deal with this situation (which are frequent in VANETs due to network partitions) in BRAVE we adopt a store-carry-forward approach. That 
is, we use a packet buffer to store the data packet until a new neighbor being better than the current node shows up. Note that these neighbors are discovered by receiving their periodic beacon messages, but information contained in beacons is not used to take forwarding decisions. The reason why beacons are not fully eliminated is because they are mandatory in the current IEEE 802.11p and DSRC standards under development. So, they would be present anyways.

\section{Improved data forwarding by adjusting waiting times}

If we adjust properly the waiting times that nodes select before sending their RESPONSE message we can reduce the overhead of the protocol and increase its reliability. In particular, we aim at getting as few responses as possible by making better candidates to answer first and cancel the responses of other neighboring vehicles. For the sake of simplicity, we assume without loss of generality the case in which we are using as routing metric the distance to destination. That is, from all neighbors the best one would be the one being closer to the next junction. The same can be done for other metrics (e.g. remaining time, delay, etc.) by just normalizing the values between a minimum and a maximum waiting time.

To adjust timers based on the goodness of candidates, we define the progress that a neighbor $n$ provides for a message addressed towards a junction $d$ with respect to the current forwarder $c$ as:

$$
P(n, d, c)=\operatorname{dist}(c, d)-\operatorname{dist}(n, d)
$$

where $\operatorname{dist}(a, b)$ represents the Euclidean distance between the position of the nodes $a$ and $b$.

The larger the progress provided toward the next junction by a neighboring vehicle, the smaller the waiting time should be. We define the Forwarder Coverage Area (FCA) as the circle with center in the current forwarder and radius the theoretical radio range $(r)$. Note that this radius is used as a reference but the protocol can work in situations in which the actual radio range is different from $r$. We divide the FCA into a number subareas of equal width. This is depicted in Fig. 2. We then assign waiting times so that all nodes in the same sub-area get the same waiting time, which is then modified by a random component to prevent collisions across nodes in that same area. The assignment function will be defined in such a way that the waiting times associated to each area are ordered according to their progress. Given a Number of Sub Areas (NSA) in which the FCA is divided, a node can easily compute the Common Sub Area (CSA) in which it is located

$$
C S A=\left\lfloor N S A \times \frac{r-P(n, d, c)}{2 r}\right\rfloor
$$

The value of CSA is an integer between 0 and $N S A-1$ corresponding 0 to the area which provides the larger progress. Once the CSA is calculated, each vehicle can compute its waiting time as

$$
T=\left(C S A \times \frac{T m a x}{N S A}\right)+\operatorname{random}\left(\frac{T \max }{N S A}\right)
$$

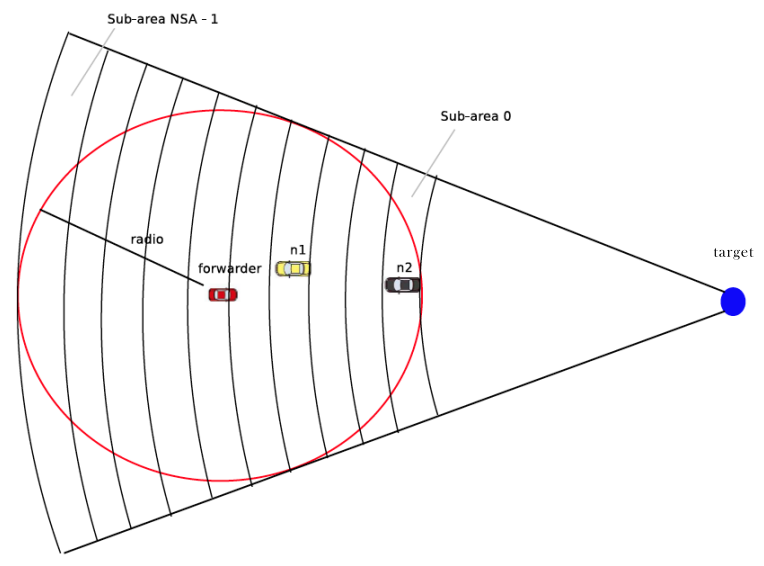

Fig. 2. Division in areas

where Tmax is the maximum time that the forwarder waits until receiving a RESPONSE message from any neighbor. The function $\operatorname{random}(x)$ returns a randomized value between 0 and $x$.

By using this discrete function we ensure that vehicles from areas closer to the current forwarder only generate RESPONSE messages if there are not better vehicles in the subareas providing more progress. In addition, the random component prevents collisions among vehicles located in the same sub-area.

\section{iII. Performance Evaluation}

To assess the performance of BRAVE, we have conducted a set of simulation experiments comparing the performance of existing VANET routing protocols in a realistic scenario. Below we give the details of the simulations and analyze the main results.

\section{A. Simulation Setup}

In order to evaluate the performance of our proposed solution, we have implemented GSR, SAR, A-STAR, GPCR, GeOpps and BRAVE protocols within The Network Simulator $n s-2$, version $2.33^{1}$. To generate the simulation scenario (street map) and the vehicular mobility patterns, we have used the well-known SUMO tool ${ }^{2}$. In particular, we have run our simulations in an area of $5 \times 4 \mathrm{~km}^{2}$ in the city center of Murcia, Spain. We have selected the most relevant streets, so our scenario consists of 53 streets and 28 junctions. Vehicles move through 20 predefined routes at a maximum speed of $50 \mathrm{~km} / \mathrm{h}$ inside the city, and $80 \mathrm{~km} / \mathrm{h}$ on the highway that crosses the scenario. The routes followed by the vehicles have been selected according to realistic situations. We have also considered a wide range of traffic densities. Vehicles are injected into its route at a certain traffic rate. This rate is varied from $1 / 30$ to $1 / 10$ vehicles per second. In such a manner the $1 / x$ rate means that each $x$ seconds a new vehicle is injected into its route.

\footnotetext{
${ }^{1}$ http://www.isi.edu/nsnam/ns/

${ }^{2}$ http://sumo.sourceforge.net/
} 
In our simulations, wireless signals propagate according to the two-ray-ground model. Vehicles carry out their communications via $802.11 \mathrm{p}$ interface card, implementing the enhanced $n s-2802.11$ physical and MAC models ${ }^{3}$. Transmission power is adjusted allowing a maximum transmission range of $250 \mathrm{~m}$.

We have simulated 10 independent runs for each configuration (figures in section III-B show the average of such runs). For each run, there are 100 different random data sources. Each one sends a 512 bytes data message towards a static station located in the center of the city. This is done to avoid introducing bias in our comparison due to considering different moving vehicles as destinations. We have employed a beacon interval of 2 seconds. Since the protocols employ geographic routing, each vehicle needs to know the position of the destination. For a fair comparison we have assumed that this information is known by all vehicles although they would use a location service in practice. Our implementation of GPCR computes the correlation coefficient of the neighbors when a vehicle checks whether it is a coordinator or not. In the case of GSR, the list of junctions that a data message must traverse is included within the message header. The same criterion is applied to SAR, since it is strongly based on GSR. Moreover, SAR uses a message buffer to store messages that can not be forwarded at a particular moment towards the destination. Each message can be held in the packet buffer for 30 seconds. To be fair, we have not enriched any of the protocols with any additional information (e.g. street density) other than the city map. For A-STAR we implement its recovery strategy when a packet gets stuck in a local minimum. Finally, in the case of GeOpps, the useful lifetime before data messages get discarded has been set to 180 seconds.

\section{B. Analysis of results}

To assess the performance of the different protocols, we consider the packet delivery ratio (PDR) and the end-toend delay. In addition to that, we also perform a detailed study of the causes of packet drops to better understand the effectiveness of BRAVE to deal with those issues.

Figure 3 shows the PDR achieved by each protocol as the density of vehicles increase. We can see that BRAVE outperforms all other protocols, obtaining a delivery ratio around 0.8 and 0.9 for all evaluated densities. GeOpps is also sensibly better than the other schemes but this comes at the price of additional delay as we shall explain later on. We can see that the protocols based on geographic routing show a "bell-shaped" PDR graph. The reason is that for low densities the PDR is low because the network is highly disconnected. For high densities the PDR gets low again due to the high contention and transmission failures at the MAC layer. In particular, the "Range Limit Problem" [14] that we mentioned in the previous section. This occurs because the higher the density, the higher the probability of finding a neighbor just near the transmission range of a vehicle. Therefore, its is more likely to choose a neighbor which has a low probability

\footnotetext{
${ }^{3}$ http://dsn.tm.uni-karlsruhe.de/english/Overhaul_NS-2.php
}

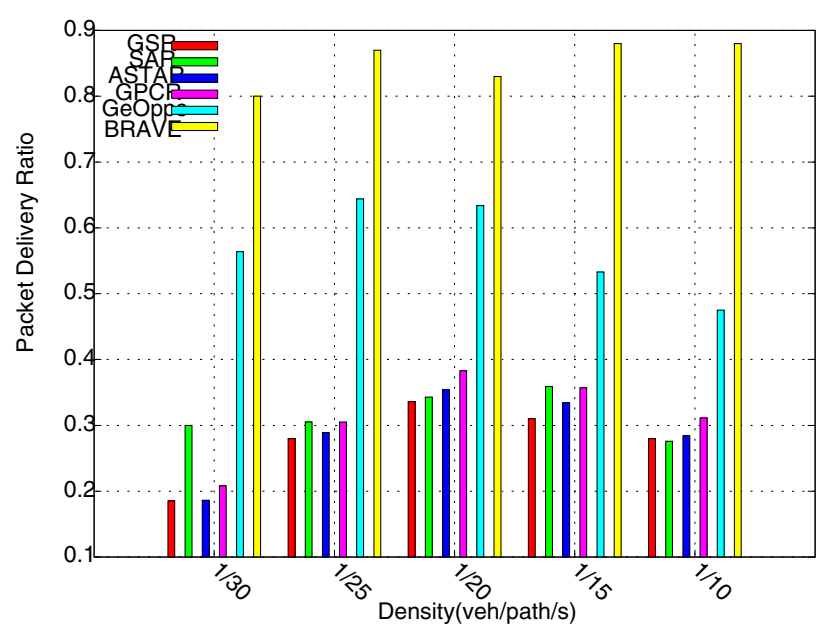

Fig. 3. Packet Delivery Ratio

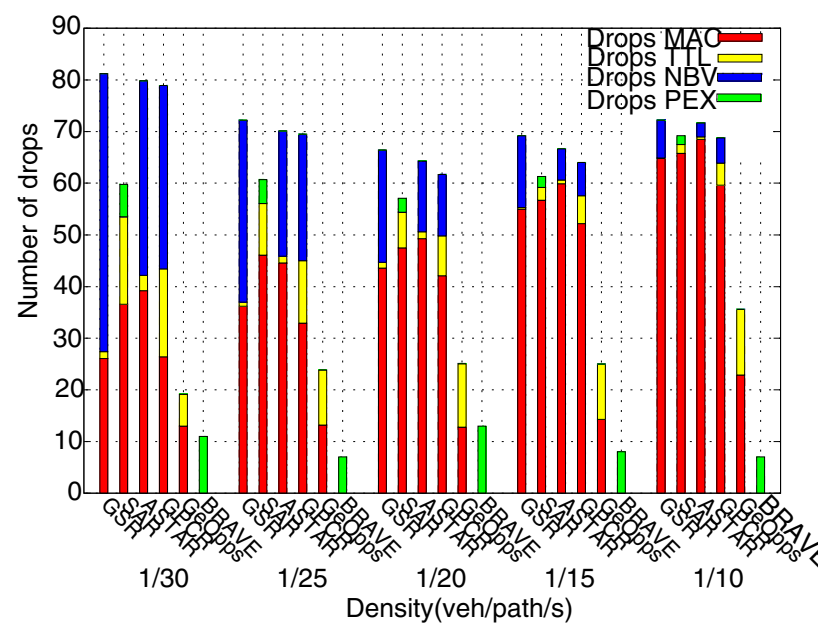

Fig. 4. Analysis of the cause of drops

of reception without errors. In contrast, BRAVE solves that problem thanks to its opportunistic next hop selection. In fact, the higher the traffic density the higher the delivery rate it obtains. When the density is low and there are no neighbors, BRAVE stores the packet until any new neighbor appear into the transmission range of the forwarder vehicle, and the packet is forwarded. This is the reason why BRAVE obtains good results across a wide range of vehicle densities.

This is clearly supported by analysis of the cause of packet drops shown in Fig. 4. We can see how for low densities the other geographic protocols drop packets mainly due to not finding appropriate neighbors (NBV) and when density increases it is mainly due to the "Range Limit Problem" (MAC). In the case of BRAVE not only there are only a few drops, but also that the protocol is not affected by the "Range Limit Problem" because the opportunistic next hop selection works remarkably well.

GeOpps is not very severely affected since it does not employ geographic routing. Messages are only handed over from one vehicle to another if the route of the other vehicle 


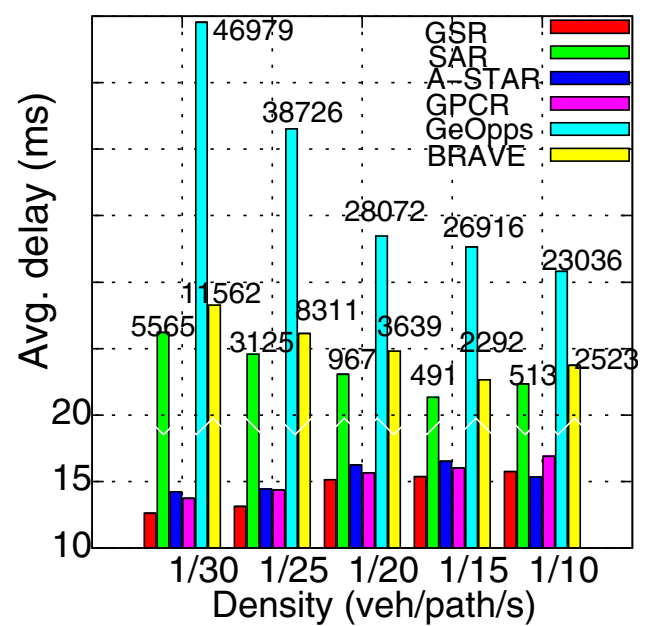

Fig. 5. End-to-end Delay

provides a better expected delivery time. So, there are fewer communications over lossy links which ends up in having a slightly better reliability at the expense of additional delay. The losses in GeOpps are mainly due to exceeded TTL (packets that exceed the maximum hop count). This is a documented problem. (see [14] for additional details).

Finally, we study the end-to-end delay in Fig. 5. As we can see, those protocols that count on a buffer to deal with temporary network disconnections present additional delay, but at the same time they are able to deliver messages in scenarios with large disconnections in which the other protocols just fail. As the density of vehicles increases, the network is more connected and allows protocols to reduce the end-to-end delay. By being delay tolerant, GeOpps is the one with larger delays. This is because the message is forwarded only to vehicles which can carry the message closer to the destination. In the case of BRAVE, the proposed protocol manages to get reasonable delays when the network is connected, while still being able to deliver messages in disconnected networks at the expense of higher delays. The other protocols have a much shorter delay, not because they are better in finding paths, but due to the fact that they only manage to deliver the messages when the network is well-connected and the destination is not very far from the source.

\section{CONCLUSiOnS AND Future Work}

In this paper we analyze the problem of efficient routing in vehicular networks. This is a challenging problem because of the intrinsic properties of VANETs such as frequent disconnections, variable topology, constrained mobility, etc.

We present a novel algorithm called BRAVE (Beacon-less Routing Algorithm for Vehicular Environments). The main ideas behind BRAVE are an opportunistic next hop selection within the routing process, and an improved overall path re-computation. The opportunistic neighbor selection allows the protocol to obtain a high reliability in relatively dense scenarios. The proposed schemes eliminates a lot of contention and guarantees that the next hop has successfully received the data packet. The second novelty refers to the fact that BRAVE allows intermediate nodes to recompute the trajectory (list of streets) towards the destination. Just by adding the positions of the next two corners the protocol is able to effectively follow the best paths while still working with local information.

Our simulation results show that the proposed protocol is able to outperform existing solutions in terms of PDR over a wide range of vehicle densities. In addition, it manages to get a good tradeoff between delivery ratio and end-to-end delay.

For future works we intend to evaluate the performance of the protocol for different amounts of available additional information (e.g. densities of cars along streets, etc.) as well as evaluating its performance with other routing metrics beyond hop count.

\section{REFERENCES}

[1] H. Moustafa and Y. Zhang, Vehicular Networks: Techniques, Standards, and Applications. Boston, MA, USA: Auerbach Publications, 2009, ch. 5 .

[2] F. Li and Y. Wang, "Routing in Vehicular Ad Hoc Networks: A Survey," 2007, IEEE Vehicular Technology Magazine.

[3] C. Lochert, M. Mauve, H. Füßler, and H. Hartenstein, "Geographic Routing in City Scenarios," ACM SIGMOBILE Mobile Computing and Communications Review, vol. 9, no. 1, pp. 69-72, 2005.

[4] V. Naumov and T. Gross, "Connectivity-aware routing (car) in vehicular ad-hoc networks," in Proc. 26th IEEE International Conference on Computer Communications (INFOCOM '07), Anchorage, Alaska, USA, May 2007, pp. 1919-1927.

[5] Christian Lochert, Hannes Hartenstein, Jing Tian, Holger Füßler, Dagmar Hermann, and Martin Mauve, "A Routing Strategy for Vehicular Ad Hoc Networks in City Environments," Junio 2003, pp. 156-161, in Proceedings of the IEEE Intelligent Vehicles Symposium 2003.

[6] J. Tian, L. Han, K. Rothermel, and C. Cseh, "Spatially Aware Packet Routing for Mobile Ad Hoc Inter-Vehicle Radio Networks," Octubre 2003, pp. 1543-1551, in Proceedings of the IEEE Intelligent Transportation System Conference.

[7] M. Jerbi, R. Meraihi, S.-M. Senouci, and Y. Ghamri-Doudane, "Gytar: improved greedy traffic aware routing protocol for vehicular ad hoc networks in city environments,' in VANET '06: Proceedings of the 3rd international workshop on Vehicular ad hoc networks. New York, NY USA: ACM, 2006, pp. 88-89.

[8] B.C. Seet, G. Liu, B.S. Lee, C.H. Foh, K.J. Wong, and K.K. Lee, "A Mobile Ad Hoc Routing Strategy for Metropolis Vehicular Communications," Diciembre 2004, pp. 989-999, in Proceedings of 3rd International Networking Conference IFIP-TC6.

[9] H. Wu, R. Fujimoto, R. Guensler, and M. Hunter, "Mddv: a mobilitycentric data dissemination algorithm for vehicular networks," in VANET '04: Proc. of the 1st ACM international workshop on Vehicular ad hoc networks, 2004, pp. 47-56.

[10] J. Zhao and G. Cao, "Vadd: Vehicle-assisted data delivery in vehicular ad hoc networks," in IEEE INFOCOM'06, 2006.

[11] Y. Ding, C. Wang, and L. Xiao, "A static-node assisted adaptive routing protocol in vehicular networks," in VANET '07: Proc. of the fourth ACM international workshop on Vehicular ad hoc networks, 2007, pp. 59-68.

[12] J. Lebrun, C.-N. Chuah, and D. Ghosal, "Knowledge-based opportunistic forwarding in vehicular wireless ad hoc networks," in Vehicular Technology Conference, 2005. VTC 2005-Spring. 2005 IEEE 61st, vol. 4, 2005, pp. 2289-2293.

[13] I. Leontiadis and C. Mascolo, "GeOpps: Opportunistic Geographical Routing for Vehicular Networks," 2007, in Proceedings of the IEEE Workshop on Autonomic and Opportunistic Communications.

[14] Victor Cabrera and Fran J. Ros and Pedro M. Ruiz, "Simulation-based Study of Common Issues in VANET Routing Protocols," Barcelona, Spain, April 2009, in Proceedings IEEE 69th Vehicular Technology Conference (VTC2009-Spring 2009).

[15] Juan A. Sánchez and Rafael Marin-Pérez and Pedro M. Ruiz, "BeaconLess Geographic Routing in Real Wireless Sensor Networks," Journal of Computer Science and Technology, vol. 23, no. 3, pp. 438-450, May 2008. 